\title{
RESEARCH
}

Open Access

\section{Hypoxia alleviates dexamethasone-induced inhibition of angiogenesis in cocultures of HUVECs and rBMSCs via HIF-1a}

Miaomiao Chai, Ce Gu, Qihua Shen, Jiaxing Liu, Yi Zhou, Ziyang Jin, Wanli Xiong, Yan Zhou* (D) and Wensong Tan

\begin{abstract}
Background and aim: Inadequate vascularization is a challenge in bone tissue engineering because internal cells are prone to necrosis due to a lack of nutrient supply. Rat bone marrow-derived mesenchymal stem cells (rBMSCs) and human umbilical vein endothelial cells (HUVECS) were cocultured to construct prevascularized bone tissue in osteogenic induction medium (OIM) in vitro. The angiogenic capacity of HUVECs was limited in the coculture system. In this study, the effects of the components in the medium on HUVEC angiogenesis were analyzed.
\end{abstract}

Methods: The coculture system was established in OIM. Alizarin red staining and alkaline phosphatase staining were used to assess the osteogenic ability of MSCs. A Matrigel tube assay was used to assess the angiogenic ability of HUVECs in vitro. The proliferation of HUVECs was evaluated by cell counting and CCK-8 assays, and migration was evaluated by the streaked plate assay. The expression levels of angiogenesis-associated genes and proteins in HUVECs were measured by qRT-PCR and Western blotting, respectively.

Results: Dexamethasone in the OIM suppressed the proliferation and migration of HUVECs, inhibiting the formation of capillary-like structures. Our research showed that dexamethasone stimulated HUVECs to secrete tissue inhibitor of metalloproteinase (TIMP-3), which competed with vascular endothelial growth factor (VEGF-A) to bind to vascular endothelial growth factor receptor 2 (VEGFR2, KDR). This effect was related to inhibiting the phosphorylation of ERK and AKT, which are two downstream targets of KDR. However, under hypoxia, the enhanced expression of hypoxiainducible factor-1a (HIF-1a) decreased the expression of TIMP-3 and promoted the phosphorylation of KDR, improving HUVEC angiogenesis in the coculture system.

Conclusion: Coculture of hypoxia-preconditioned HUVECs and MSCs showed robust angiogenesis and osteogenesis in OIM, which has important implications for prevascularization in bone tissue engineering in the future.

Keywords: Mesenchymal stem cell, Human umbilical vein endothelial cell, Angiogenesis, Dexamethasone, Hypoxia

\footnotetext{
* Correspondence: zhouyan@ecust.edu.cn

State Key Laboratory of Bioreactor Engineering, School of Bioengineering, East China University of Science and Technology, 130 Meilong Rd, Shanghai 200237, People's Republic of China
} 


\section{Introduction}

During the past two decades, bone tissue engineering techniques have offered promising alternative approaches for the treatment of critical bone defects [1]. Cell-based constructs have received much attention in combining bone repair with stem cells. However, cell-based constructs with insufficient vascular networks may be prone to necrosis due to a lack of nutrients, oxygen, and cytokines [1-3] and would delay the bone repair process. In general, prevascularization strategies in vivo and in vitro are two main methods of tissue engineering construction [4]. Prevascularization in vitro is mainly based on the coculture of human umbilical vein endothelial cells (HUVECs) with mesenchymal stem cells (MSCs). The formation of a capillary-like network has been observed in the coculture system with growth medium [5-7]. However, our previous research found that HUVECs promoted the osteogenesis of MSCs, while the angiogenesis of HUVECs was inhibited in OIM [8].

The osteogenic environment is associated with differentiated MSCs, HUVECs, and OIM. Studies have shown that many factors have a negative effect on the angiogenesis of HUVECs, including differentiated MSCs and OIM [8-10]. However, it is still unknown which components in OIM inhibit the angiogenesis of HUVECs. OIM consists of Dulbecco's modified Eagle's medium (DMEM), $\beta$-glycerophosphate disodium ( $\beta$-gpd), L-Vitamin $C(\mathrm{~L}-\mathrm{VC})$, and dexamethasone (DEXA). Dexamethasone is necessary in OIM and plays an indispensable role in osteogenesis. Dexamethasone promotes the expression of type I collagen, alkaline phosphatase, osteocalcin, and bone sialoprotein [11]. However, dexamethasone has been found to inhibit the formation of blood vessels in the tumor microenvironment [12] and in embryological development [13]. Dexamethasone inhibits the protein expression and activity of eNOS, which is not conducive to angiogenesis and fetal development [14]. Furthermore, dexamethasone has been proven to inhibit angiogenesis by microRNA-34a, leading to femoral head necrosis [15]. In addition, dexamethasone inhibits angiogenesis by downregulating VEGF secretion to block tumor progression [16]. However, how dexamethasone functions in the angiogenesis of HUVECs in the presence of OIM in a coculture system of MSCs and HUVECs still needs to be explored.

Vascular endothelial growth factor (VEGF) is the most important growth factor during angiogenesis. VEGF binds to vascular endothelial growth factor receptor 2 (VEGFR2, KDR), resulting in KDR phosphorylation. Phosphorylated KDR activates the downstream targets ERK and AKT, promoting the proliferation, migration, and vascular network generation of HUVECs. Tissue inhibitor of metalloproteinase (TIMP-3) is unique, endogenous protein that competes with VEGF for KDR binding in vivo [17]. TIMP-3 downregulates the phosphorylation of
KDR and its downstream signaling pathways, inhibiting the angiogenesis of HUVECs. Recent studies have shown that dexamethasone can stimulate TIMP-3 expression in murine fibroblasts [18]. After administration of dexamethasone in rats, the gene expression level of TIMP-3 in the lungs was doubled compared with that in the dexamethasone-free group [19]. Furthermore, numerous studies have shown that hypoxia can downregulate TIMP-3 expression in retinal epithelial cells [20].

Hypoxia in vivo is beneficial for angiogenesis, which promotes bone repair [21]. On the one hand, hypoxia can modulate glycolysis metabolism (glucose transporter $1 / 3$, hexokinase $1 / 3$, lactate dehydrogenase, and pyruvate kinase $M$ ) to mediate postimplantation cell survival before the formation of blood vessels [22]. On the other hand, hypoxia-inducible factor (HIF-1 $\alpha)$, which is expressed in hypoxic conditions, can promote blood vessel formation, which affects the transport of oxygen and nutrients to internal cells, by increasing the expression of a large number of proangiogenic factors. Therefore, hypoxia seems to be an effective approach to improve angiogenesis in bone tissue constructors in vitro.

To improve the angiogenesis of HUVECs in the presence of OIM in the coculture system, the role of dexamethasone in the inhibition of angiogenesis in the OIM coculture system still needs to be explored. Cobalt chloride $\left(\mathrm{CoCl}_{2}\right)$ stimulated hypoxia was used to alleviate dexamethasoneinduced inhibition of HUVEC angiogenesis. The results reveal the behavior and internal mechanism of HUVECs in OIM under a coculture system and provide a strategy for prevascularization in bone tissue engineering in the future.

\section{Materials and methods}

\section{Cell isolation and cell culture}

All procedures on rats were performed in full compliance with the guidelines of the Ethics Committee at East China University of Science and Technology. Four-week-old Sprague Dawley rats (Shanghai SLAC Laboratory Animal Co., Ltd), which were SPF grade, male and weighed $\sim 80-120 \mathrm{~g}$, were used. BMSCs were isolated using the bone marrow adherence method. Cells were cultured in $\alpha$ minimum essential medium ( $\alpha$-MEM, Gibco, USA) supplemented with $10 \%$ fetal bovine serum (FBS, HyClone, USA) at $37^{\circ} \mathrm{C}$ in a $5 \% \mathrm{CO}_{2}$ humidified atmosphere. The medium was changed every 2 days. Cells at passages 3 to 5 were used in the experiments.

Primary HUVECs were purchased from ScienCell and cultured in endothelial cell growth medium (ECM, ScienCell, USA) containing 5\% FBS and supplemented with 100x endothelial cell growth supplement (ECGS, ScienCell, USA). The medium was changed every 2 days. Cells at passages 3 to 8 were used in the experiments. 


\section{Osteogenic differentiation assay}

MSCs were seeded in 24-well plates at a density of $1 \times 10^{4}$ cells/well in growth medium. After $24 \mathrm{~h}$, the medium was changed to OIM that comprised DMEM (Gibco, USA) supplemented with $10 \% \mathrm{FBS}, 10^{-7} \mathrm{M}$ dexamethasone (Sigma, USA), $10 \mathrm{mM} \quad \beta$-glycerophosphate disodium (Sigma, USA), and $50 \mu \mathrm{g} / \mathrm{ml} \mathrm{L-Vc} \mathrm{(Sigma).} \mathrm{The} \mathrm{medium}$ was replaced every 3 days. After induction, mineralized nodules were detected by alizarin red $\mathrm{S}$ staining (ARS, Sigma). Alkaline phosphatase (ALP) enzymatic activity was analyzed using an NBT/BCIP alkaline phosphatase color development kit (Beyotime, China).

For coculture of MSCs and HUVECs, MSCs were seeded in 24-well plates at a density of $1 \times 10^{4}$ cells/well, while HUVECs were seeded at a density of $2 \times 10^{4}$ cells/ well. The initial medium was a 1:1 (v/v) mixture of $\alpha$ MEM and ECM supplemented with 10\% FBS and 100x ECGS. After $24 \mathrm{~h}$, the medium was changed to OIM supplemented with 100× ECGS.

\section{In vitro tube formation assay}

HUVEC monoculture or coculture with MSCs was performed on growth factor-depleted Matrigel (BD, USA) in 96-well plates with $50 \mu \mathrm{L}$ of Matrigel/well. The capillary-like structure was viewed $6 \mathrm{~h}$ later. Microscopic fields containing the tube structure that formed on the gel were photographed using fluorescence inverted phase contrast microscopy. Five fields per test condition were examined. The tube length was calculated using ImageJ software.

\section{In vitro migration assay}

HUVECs were seeded in 24-well plates at a density of $1 \times 10^{4}$ cells/well. After the cells reached confluence, serum deprivation was performed for $16 \mathrm{~h}$. A straight line was drawn in the bottom well with a sterile pipette tip $(200 \mu \mathrm{L}$ yellow tip). PBS was used to wash away the floating cells, and conditioned medium was added. After incubation for $6 \mathrm{~h}$, the cell migration ability was assessed by photographing the healing of the scratches using fluorescence inverted phase contrast microscopy.

\section{Cell viability assay}

HUVECs were seeded in 24-well plates at a density of $1 \times 10^{4}$ cells/well with $10^{-7} \mathrm{M}$ dexamethasone in ECM supplemented with 5\% FBS and 100× ECGS (EGM) or OIM. The medium was replaced every 2 days. The cells were incubated at $37{ }^{\circ} \mathrm{C}$ in a $5 \% \mathrm{CO}_{2}$ humidified atmosphere, digested with trypsin, and counted every day.

To determine the influence of hypoxia on HUVEC proliferation, $\mathrm{CoCl}_{2}$ (Aladdin, China) was used as a mimic for hypoxia. Cells were seeded in 96-well plates at a density of $0.5 \times 10^{4}$ cells/well in EGM containing dexamethasone with various concentrations of $\mathrm{CoCl}_{2}(0,50$,
$100,150,200$, or $250 \mathrm{mM})$. After $24 \mathrm{~h}$ or $48 \mathrm{~h}$, the cells were washed with PBS, and $200 \mu \mathrm{L}$ of $\alpha$-MEM comprising $10 \%(\mathrm{v} / \mathrm{v})$ cell counting kit-8 (CCK-8, DOJINDO, Japan) solution was added to each well, and then the plate was incubated at $37^{\circ} \mathrm{C}$ for $2 \mathrm{~h}$. The absorbance at $450 \mathrm{~nm}$ of each well was measured by an ELx800 ELISA microplate reader (BioTek).

\section{Cell cycle analysis}

HUVECs were cultured with or without dexamethasone $\left(10^{-7} \mathrm{M}\right)$ for $72 \mathrm{~h}$ and centrifuged at $1000 \mathrm{rpm}$, and then the cell pellet was collected and washed three times with cold PBS at $4{ }^{\circ} \mathrm{C}$. Then, BMSCs were fixed in $70 \%$ cold ethanol in PBS. After $24 \mathrm{~h}$, the cell cycle was measured by a cell cycle and apoptosis analysis kit (Beyotime). Cells were analyzed on a flow cytometer (FACSCalibur, $\mathrm{BD})$.

\section{RNA isolation and qRT-PCR}

Total RNA was extracted using TRIzol reagent (Invitrogen, USA) according to the manufacturer's instructions. cDNA was obtained using MLV reverse transcriptase (Promega, USA). Quantitative real-time PCR (qRT-PCR) was performed as previously reported [23]. GAPDH was used as an endogenous control. All primers (Table 1) were obtained from Sangon Biotech Co., Ltd. The data were analyzed according to the $\Delta \Delta C \mathrm{~T}$ method.

\section{Western blotting}

HUVECs were harvested and lysed in RIPA lysis buffer containing $10 \mathrm{mM}$ protease inhibitors (phenylmethylsulfonyl fluoride, PMSF, Beyotime, China). A BCA protein assay kit (Beyotime) was used to determine the protein concentrations. Then, loading buffer (Beyotime) was added to the samples. The cellular proteins were separated by $10 \%$ SDS-polyacrylamide gel electrophoresis, and the separated proteins were electrically transferred onto PVDF membranes (Millipore, USA). Then, the membranes were blocked with $5 \%$ nonfat dry milk in TBST $(20 \mathrm{mM}$ Tris- $\mathrm{HCl} \mathrm{pH} 7.4,150 \mathrm{mM} \mathrm{NaCl}$, and $0.05 \%$ Tween-20) for $1 \mathrm{~h}$. The membranes were washed with TBST and probed with primary antibodies overnight at $4{ }^{\circ} \mathrm{C}$. The bound primary antibodies (Table 2) were detected with secondary antibodies conjugated

\begin{tabular}{lll} 
Table 1 Primer sequences for qRT-PCR & \\
\hline Gene & Forward $\left(5^{\prime}-3^{\prime}\right)$ & Reverse $\left(5^{\prime}-3^{\prime}\right)$ \\
\hline VEGF & TTCAAGCCATCCTGTGTGCC & CACCAACGTACACGCTCCAG \\
TIMP-3 & CAAGGGGCTGAACTATCGGT & TCAGGGGTCTGTGGCATTGA \\
VEGFR2 & CAGCTCACAGTCCTAGAGCG & CTGCGGATAGTGAGGTTCCG \\
HIF-1a & AGGTCTAGGAAACTCAAAACCTGA & CAGAAGTTCCTCACACGCA \\
GAPDH & AATTCCATGGCACCGTCAAG & TGGTTCACACCCATGACGAA \\
\hline
\end{tabular}


Table 2 Antibody used in the western blot analysis

\begin{tabular}{llll}
\hline Antibody/marker & Dilusion & Source & Code number \\
\hline TIMP-3 & $1: 1000$ & CST & $\# 5673$ \\
VEGF-A & $1: 1000$ & Abcam & Ab69479 \\
p-KDR & $1: 1000$ & CST & $\# 2478$ \\
KDR & $1: 1000$ & CST & $\# 2479$ \\
p-AKT & $1: 1000$ & CST & $\# 4060$ \\
AKT & $1: 1000$ & CST & $\# 4691$ \\
p-ERK & $1: 1000$ & CST & $\# 4377$ \\
ERK & $1: 1000$ & CST & $\# 4695$ \\
HIF-1a & $1: 1000$ & CST & $\# 36169$ \\
$\beta$-Actin & $1: 1000$ & CST & $\# 4970$ \\
\hline
\end{tabular}

with horseradish peroxidase (HRP, Abcam, UK) and visualized by enhanced chemiluminescence (Millipore). $\beta$-Actin was used as an internal reference.

\section{Cell transfection assay}

siRNAs targeting the TIMP-3 (5'-CCAAACACUACGCC UGCAUTT-3', 5'-AUG-CAGGCGUAGUGUUUGGTT$\left.3^{\prime}\right)$ and HIF- $1 \alpha$ genes (5'-GCUGGAGACACAA-UCAUAU TT-3', 5'-AUGCAGGCGUAGUGUUUGGTT-3') (RiboBio, Guangzhou, China) were used to specifically silence TIMP-3 and HIF- $1 \alpha$ in HUVECs, and nonspecific sequence siRNAs were used as a negative control (NC). Cells were transfected with Lipo8000 according to the manufacturer's protocol (Beyotime). After $24 \mathrm{~h}$ of siRNA transfection, the knockdown efficiency was confirmed by qRT-PCR analysis.

\section{Statistical analysis \\ All experiments were repeated at least three times. All data are presented as the means \pm standard deviations. Statistical differences were evaluated using Student's two-sided $t$ tests. Differences were considered statisti- cally significant if $p<0.05$. \\ Results \\ HUVECs promoted MSC osteogenesis in a coculture system with OIM}

To evaluate the effect of HUVECs on the osteogenic differentiation of MSCs, MSCs were cultured with or without HUVECs in OIM for 21 days. Alizarin red S staining was performed to characterize MSC osteogenesis. More mineralized nodules appeared in the coculture system on days 7, 14, and 21 than in the monoculture system (Fig. 1). These results suggest that HUVECs promote MSC osteogenesis in OIM.

\section{OIM inhibited HUVEC angiogenesis}

To observe HUVEC angiogenesis in the coculture system, a Matrigel tube formation assay was used. HUVECs alone or cocultured HUVECs and MSCs were seeded on Matrigel in EGM or OIM supplemented with

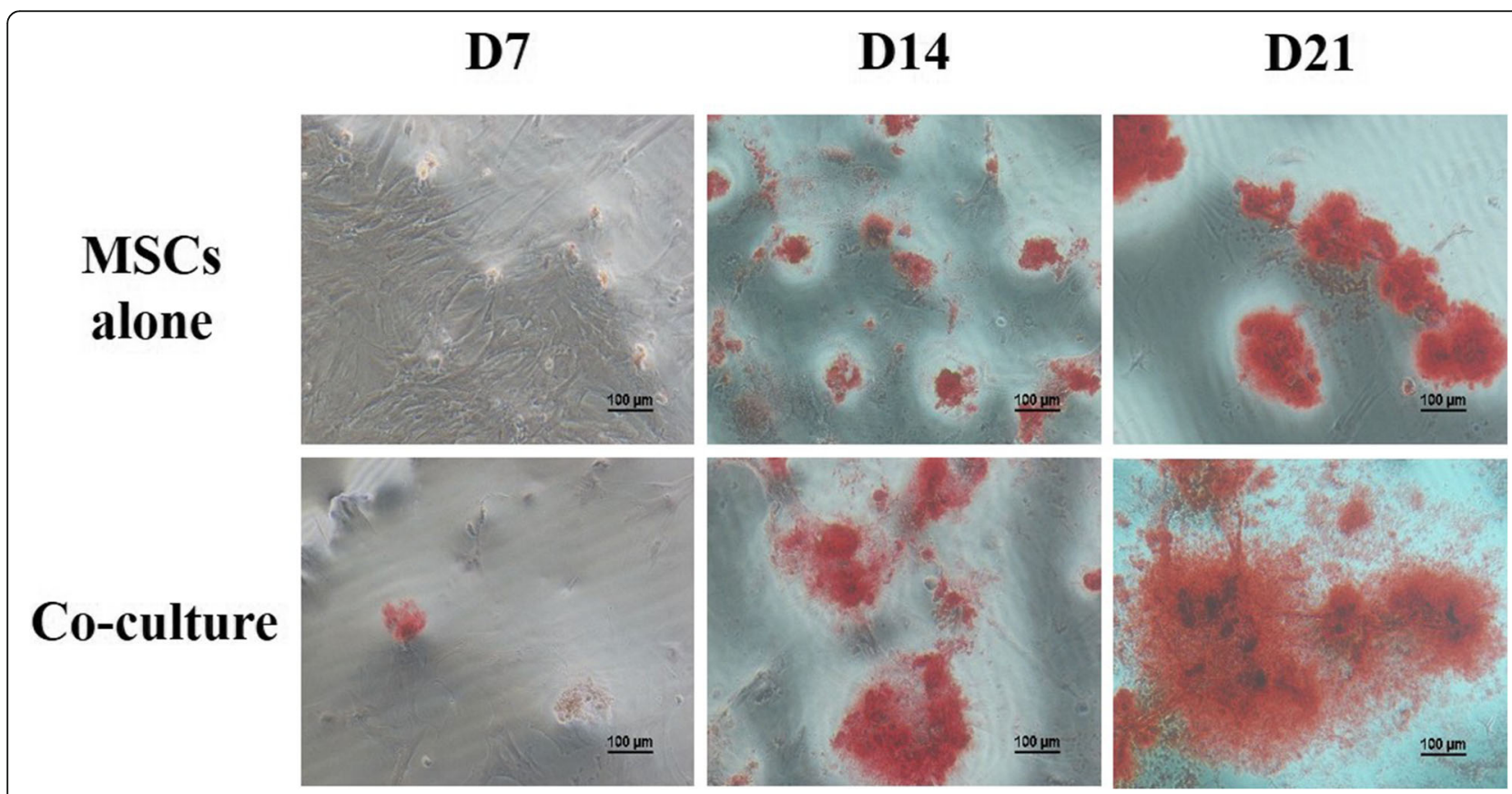

Fig. 1 HUVECS promoted MSC osteogenesis in a coculture system with OIM. MSCs were cultured in a single culture system (MSCS alone group) or a coculture system with HUVECs (coculture group). The OIM was changed every 3 days. Alizarin red S was used to detect calcium deposition on days 7, 14, and 21. Scale bar, $100 \mu \mathrm{m}$ 
ECGS and incubated for $6 \mathrm{~h}$. With or without MSCs, HUVECs failed to form capillary-like structures in OIM (Fig. 2a). The migration and proliferation capacities of HUVECs were significantly suppressed in OIM compared with EGM (Fig. 2b, c). To determine how OIM impaired cell proliferation, flow cytometry was used to examine the cell cycle phases of HUVECs. After treatment with OIM, the percentage of HUVECs in the G1 phase increased, while the percentage of cells in the $S$ phase decreased compared with that of EGM (Fig. 2d).

\section{Dexamethasone inhibited HUVEC angiogenesis}

To determine which component in OIM hampered HUVEC angiogenesis, the osteogenic components in OIM, dexamethasone, $\beta$-gpd, and L-vitamin $C$, were individually added to EGM. In the Matrigel tube assay, dexamethasone significantly inhibited the formation of capillary-like structures by HUVECs (Fig. 3a). Moreover, dexamethasone negatively affected the proliferation and migration of HUVECs (Fig. 3b, c). Subsequently, the expression of angiogenesis-related genes in HUVECs treated with or without dexamethasone was measured by qRT-PCR. The level of VEGF, a proangiogenic gene, was significantly decreased in the dexamethasone group (Fig. 3d). The level of TIMP-3, an antiangiogenic gene, was dramatically increased. There was no obvious difference in the level of $K D R$.

\section{Dexamethasone suppressed HUVEC angiogenesis by upregulating the expression of TIMP-3}

TIMP-3 is known to block KDR signaling pathways, inhibiting HUVEC angiogenesis. To confirm the gene expression results of TIMP-3, Western blotting was performed to measure the expression of TIMP-3 and angiogenesis-related proteins. The results indicated that dexamethasone significantly upregulated the protein expression of TIMP-3, while TIMP-3 was further increased in the presence of OIM (Fig. 4a). The expression of VEGF-A, which was decreased in dexamethasone-treated HUVECs, was lowest in OIM. Similarly, the level of phosphorylated KDR, which was decreased in dexamethasone-treated HUVECs, was lowest in OIM. Furthermore, the levels of phosphorylated AKT and ERK, which are KDR downstream targets and are related to the proliferation and migration of HUVECs, were also significantly downregulated.

To further validate that TIMP-3 is an important mediator of dexamethasone-induced inhibition of angiogenesis, TIMP-3 was knocked down using a specific siRNA (Fig. 4b). Silencing TIMP-3 significantly rescued the capillary-like structures of HUVECs treated with dexamethasone (Fig. 4c). The Western blot results showed that siTIMP-3 inhibited the protein expression of TIMP-3, while the expression of VEGF-A was unchanged (Fig. 4d). In addition, the phosphorylation levels of KDR, AKT, and ERK were restored. These observations suggest that dexamethasone inhibits HUVEC angiogenesis by upregulating TIMP-3 expression.

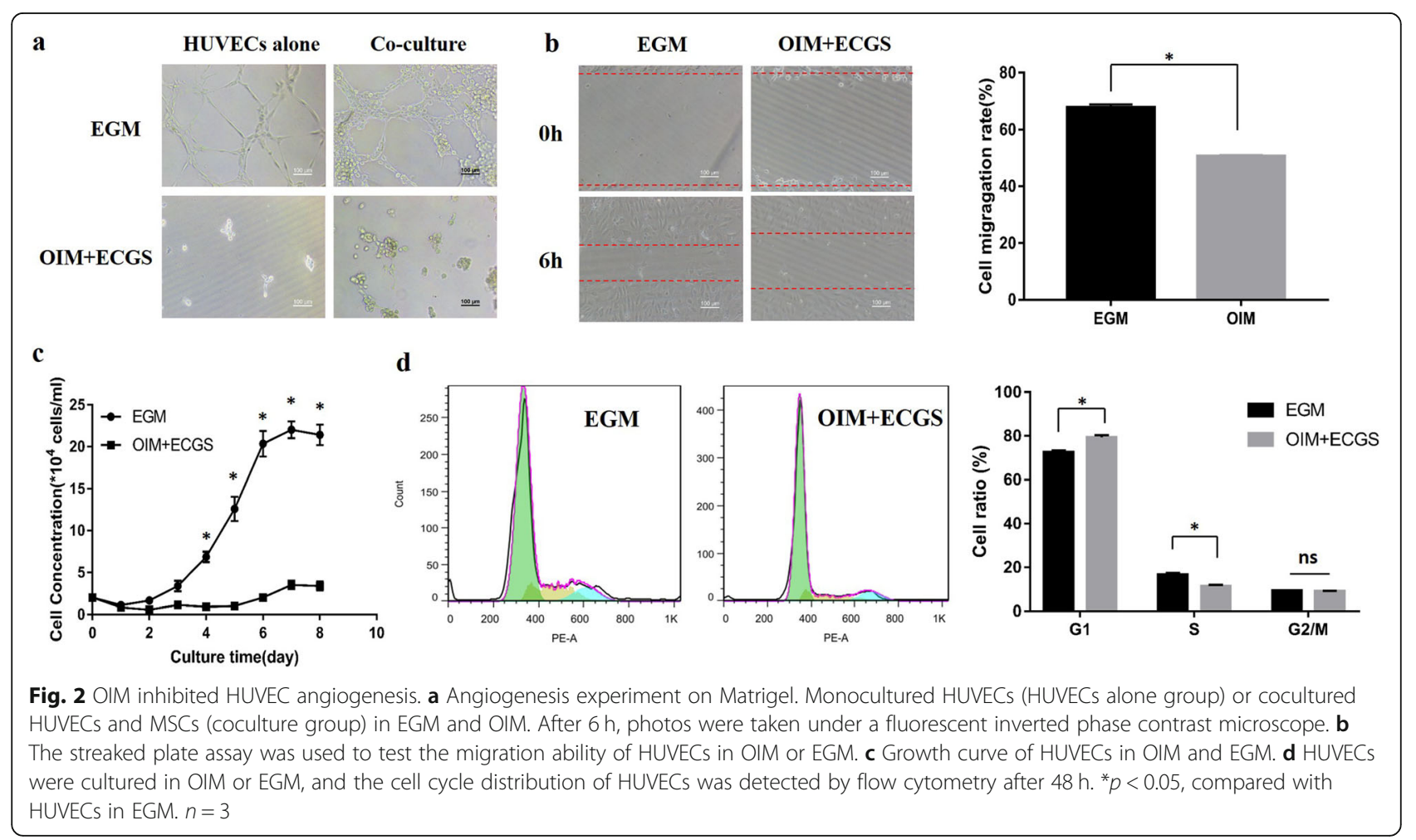




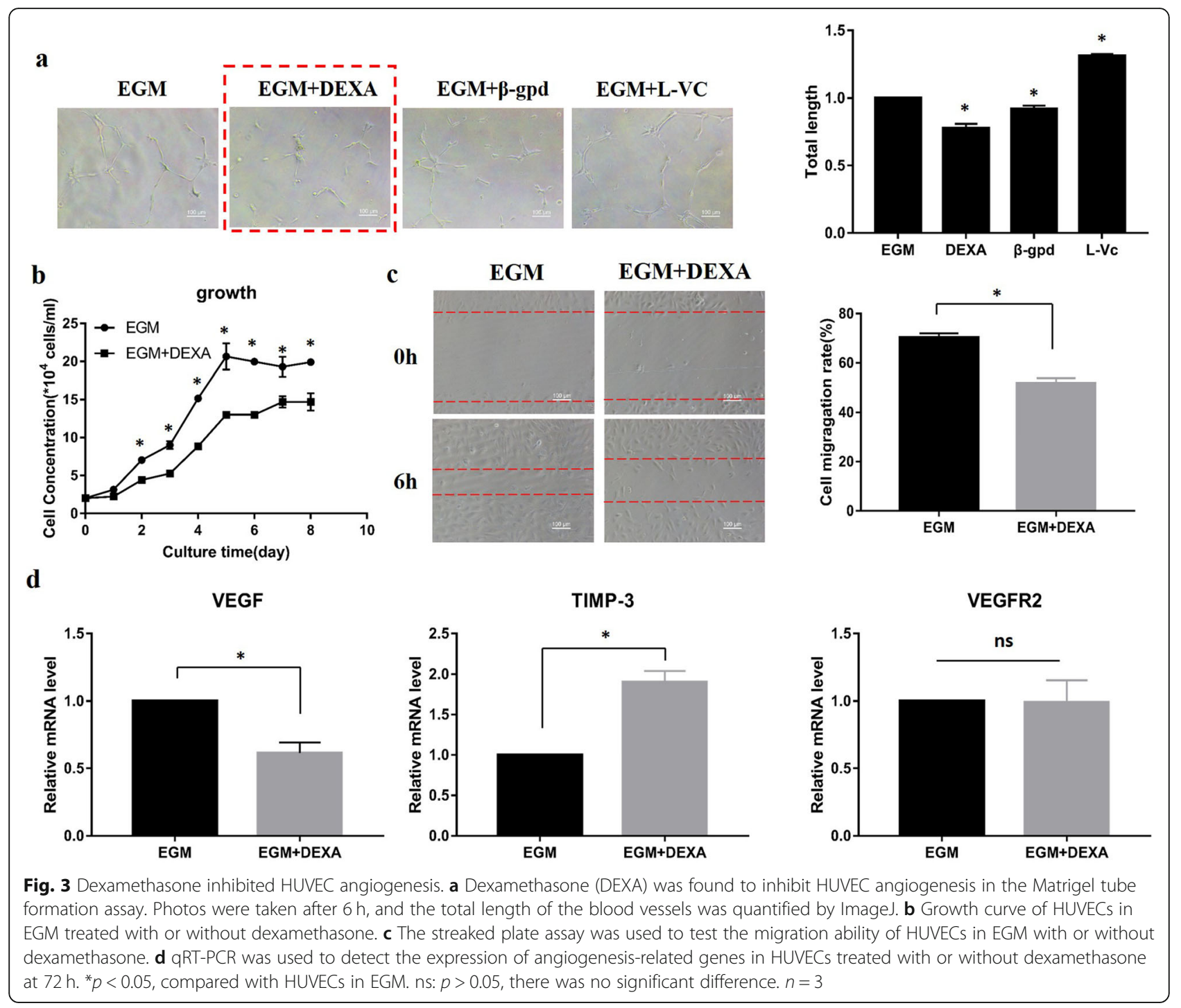

\section{Hypoxia rescued the angiogenesis of HUVECs inhibited} by dexamethasone

Studies have shown that hypoxia can stimulate angiogenesis. To determine whether hypoxia can alleviate the inhibitory effect of dexamethasone on angiogenesis, the following experiment was performed. $\mathrm{CoCl}_{2}$ was used to simulate hypoxia. $\mathrm{CoCl}_{2}(50,100,150,200$, or $250 \mu \mathrm{M})$ was added to EGM to help HUVECs form capillary-like structures on Matrigel in the presence of dexamethasone. The results suggested that both 50 and $100 \mu \mathrm{M}$ $\mathrm{CoCl}_{2}$ could significantly restore the capillary-like structures of HUVECs (Fig. 5a). Further research showed that the migration ability of HUVECs was improved in all hypoxia groups, especially in the groups treated with 50 and $100 \mu \mathrm{M} \mathrm{CoCl}_{2}$ (Fig. 5b, c). A CCK-8 assay was used to measure the proliferation capacity of HUVECs. The results suggested that HUVEC proliferation in the groups treated with 50 and $100 \mu \mathrm{M} \mathrm{CoCl}{ }_{2}$ was improved significantly at $48 \mathrm{~h}$ (Fig. 5d). Furthermore, the q-PCR results showed that the expression of VEGF-A was significantly increased, while TIMP-3 expression was decreased (Fig. 5e).

\section{Hypoxia promoted HUVEC angiogenesis via HIF-1a}

In the following experiment, $50 \mu \mathrm{M} \mathrm{CoCl}{ }_{2}$ was added to explore the mechanism by which hypoxia restores the capillary-like structures of HUVECs inhibited by dexamethasone. First, Western blotting was performed to investigate the expression of angiogenesis-related proteins under hypoxic conditions. The data showed a significant increase in the protein expression of HIF- $1 \alpha$, while TIMP-3 expression was decreased. In addition, VEGF-A protein expression was increased, and the phosphorylation level of KDR and its downstream targets AKT and ERK were significantly restored (Fig. 6a). 


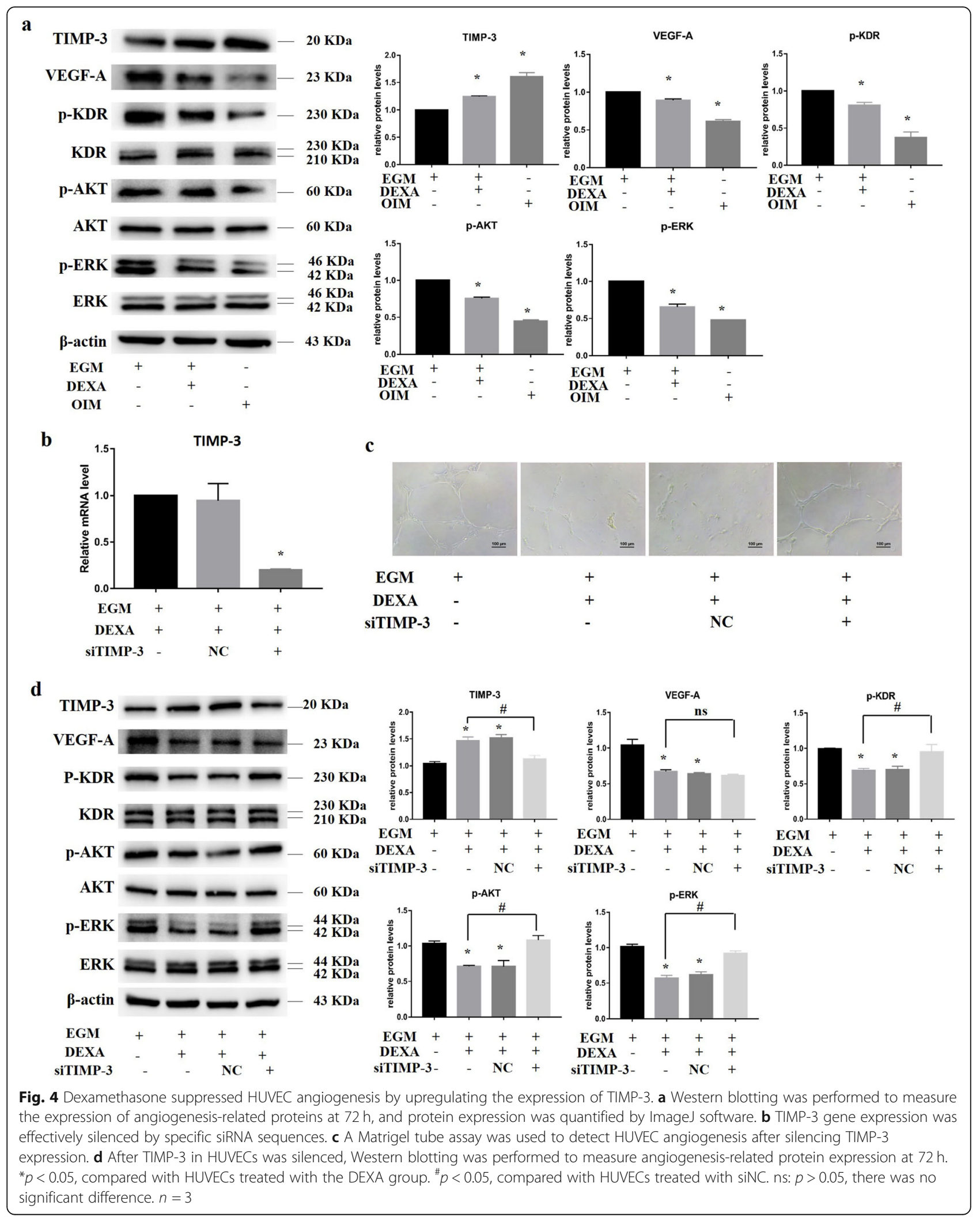




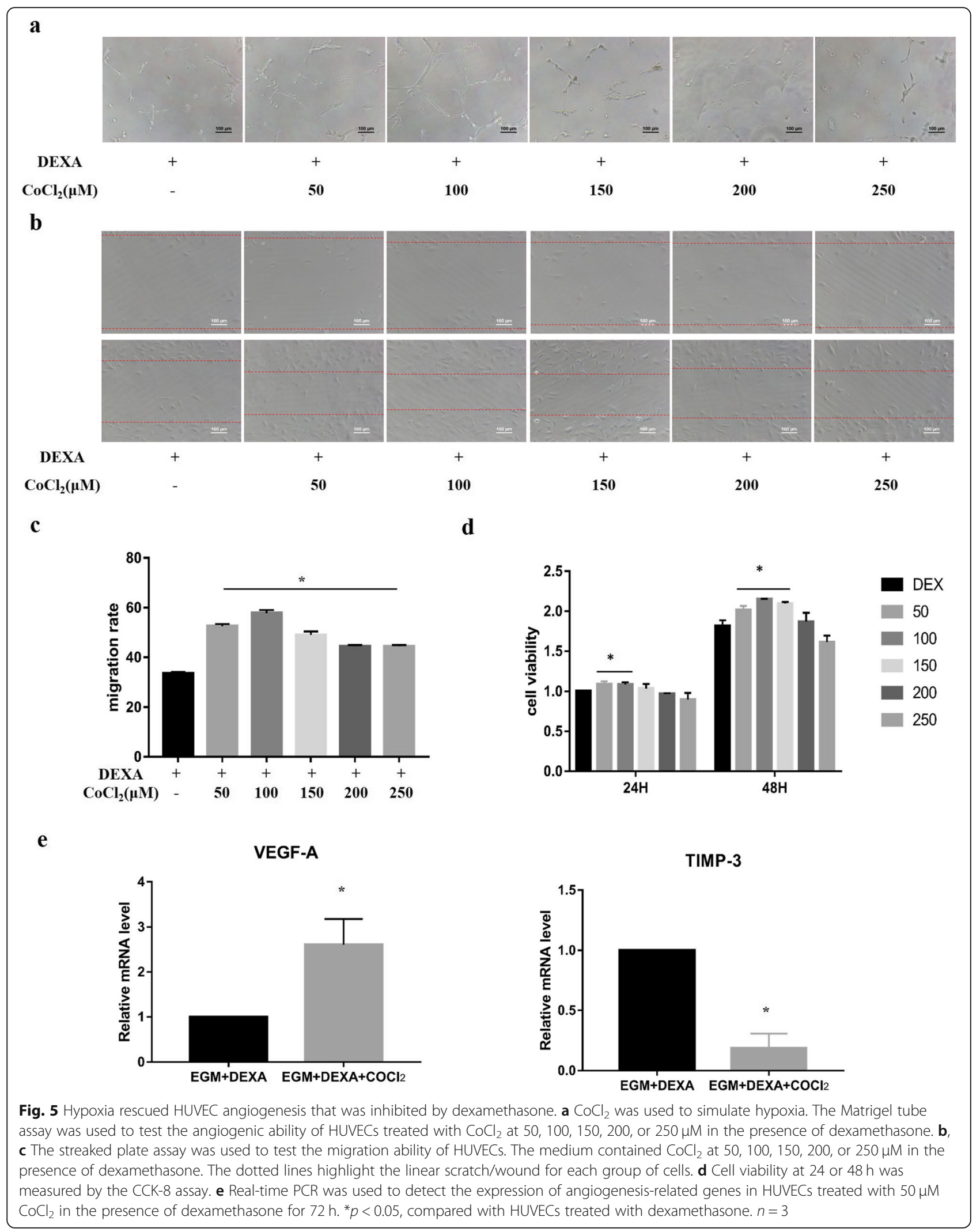




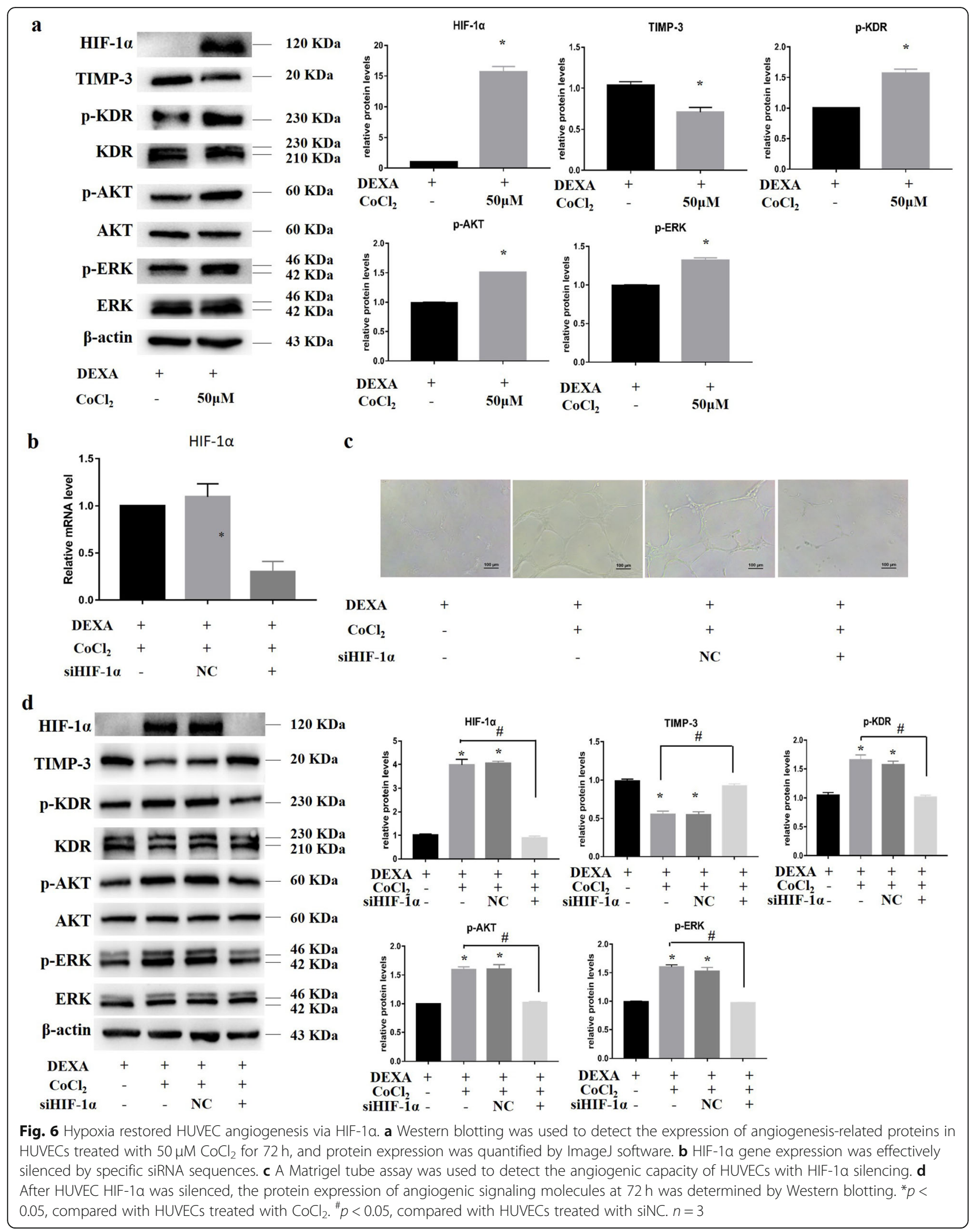


To confirm that HIF- $1 \alpha$ plays a key role in vascular restoration under hypoxic conditions, HIF-1 $\alpha$ was silenced with siRNA (Fig. 6b). Matrigel experiment results showed that hypoxia restored dexamethasoneinhibited angiogenesis, but this process was disrupted by silencing HIF-1 $\alpha$ (Fig. 6c). siRNA-mediated HIF-1 $\alpha$ gene silencing significantly decreased the protein expression of HIF- $1 \alpha$, while TIMP-3 expression was increased (Fig. 6d). The phosphorylation levels of KDR and its downstream factors AKT and ERK were also significantly decreased. These results indicate that hypoxia reduces TIMP-3 expression through HIF- $1 \alpha$, leading to increased phosphorylation of KDR and its downstream factors AKT and ERK.

\section{Hypoxia-preconditioned HUVECs were cocultured with MSCs to promote osteogenesis and angiogenesis}

We found that hypoxia-preconditioned HUVECs could promote MSCs to express ALP on day 7 and to accumulate mineralized nodules on days 14 and 21 (Fig. 7a). The Matrigel tube assay results showed that hypoxia-preconditioned HUVECs partially restored the capillary-like structures in the coculture system in the presence of OIM (Fig. 7b).

\section{Discussion}

Bone tissue engineering with HUVEC-based prevascularization could accelerate the process of bone repair. However, studies on the MSC-HUVEC coculture system with OIM showed that HUVECs promoted MSC osteogenesis, but HUVEC angiogenesis was inhibited. Further analysis showed that dexamethasone in OIM inhibited HUVEC angiogenesis.

A proposed mechanism of action is shown in Fig. 8. Dexamethasone, an indispensable component in OIM, promotes MSC osteogenesis. However, in this study, it was found that dexamethasone could stimulate HUVECs to secrete TIMP-3. This action prevented the phosphorylation of KDR and its downstream factors AKT and ERK. Subsequently, the proliferation and migration of HUVECs were inhibited, and HUVEC angiogenesis was limited. In addition, hypoxia was found to reduce the expression of TIMP-3 through HIF-1 $\alpha$. Hypoxia could alleviate dexamethasone-induced inhibition of angiogenesis in the coculture system with OIM. Moreover, hypoxiapreconditioned (HPC) HUVECs also promoted MSC osteogenesis.

VEGF-A is a key mediator of angiogenesis and mainly activates the phosphorylation of the tyrosine kinase receptor VEGFR [24]. VEGFR has three main subtype structures: VEGFR1 (Flt-1), VEGFR2 (KDR), and VEGF R3 (Flt-4). VEGFR1 and VEGFR2 mediate physiological and pathophysiological angiogenesis, while VEGFR3 mediates lymphangiogenesis. Although VEGF-A has a higher affinity for Flt-1 than KDR, KDR responds more effectively. After VEGF-A binds to KDR, the phosphorylated Y1175 residue recruits steroid receptor coactivator (Src) Homology 2 Domain Containing Adapter Protein B (SHB), activates focal adhesion kinase (FAK), and affects cell migration. The activation process of SHB also involves PI3K/AKT, which are Src substrates. AKT activates the expression of CyclinD1 via GSK-3 and mTOR to achieve cell cycle transition from G1 to $S$ phase, thus promoting cell proliferation $[25,26]$. AKT also induces cell migration via the GSK-3 $\beta / \beta$-Catenin signaling pathway [27]. In addition, Y1175 residues recruit phospholipase $\mathrm{C}$ (PLC- $\gamma)$, trigger $\mathrm{Ca}^{2+}$-dependent signaling, and activate ERK signals. ERK promotes NF- $\mathrm{KB}$ entry into the nucleus to regulate the expression of related genes,

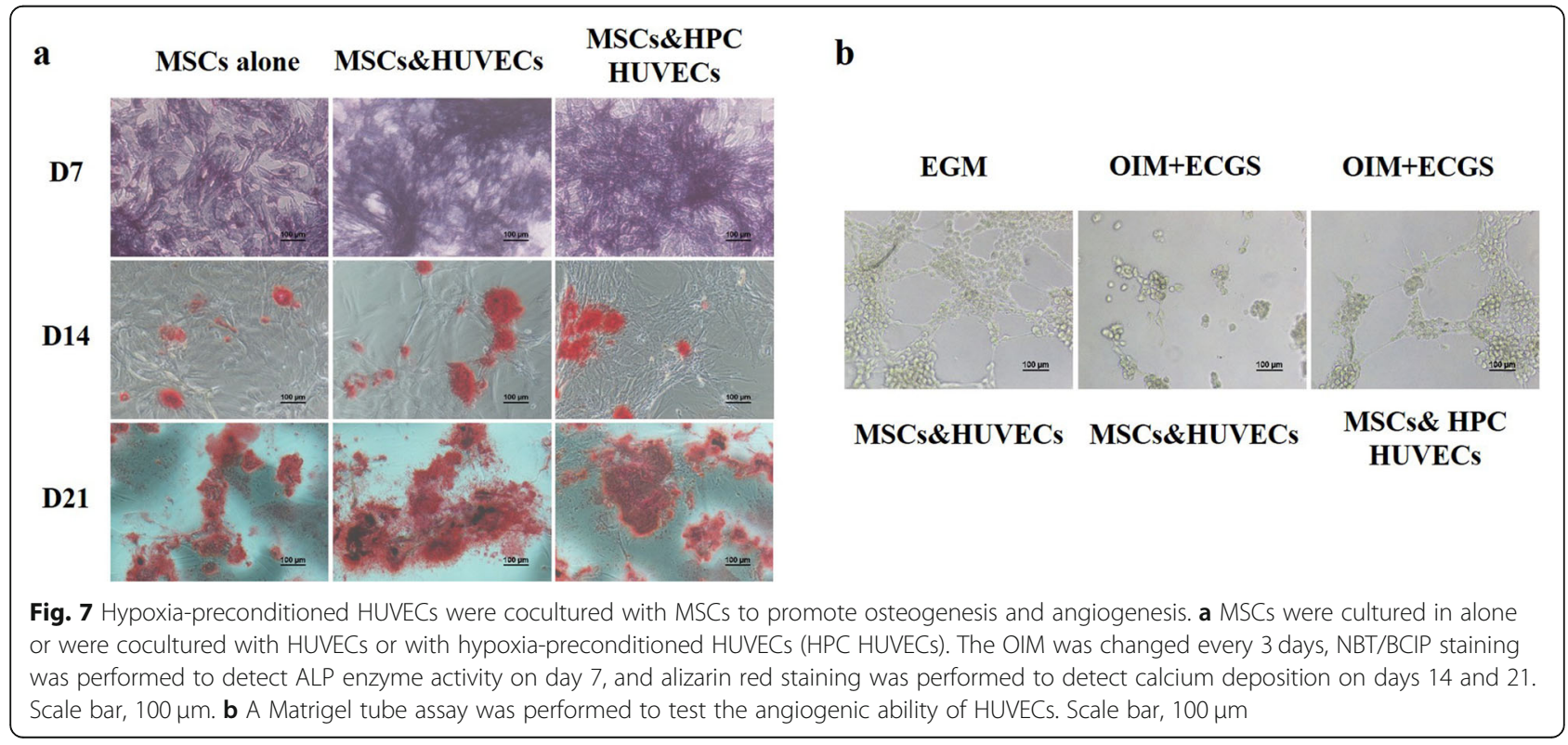




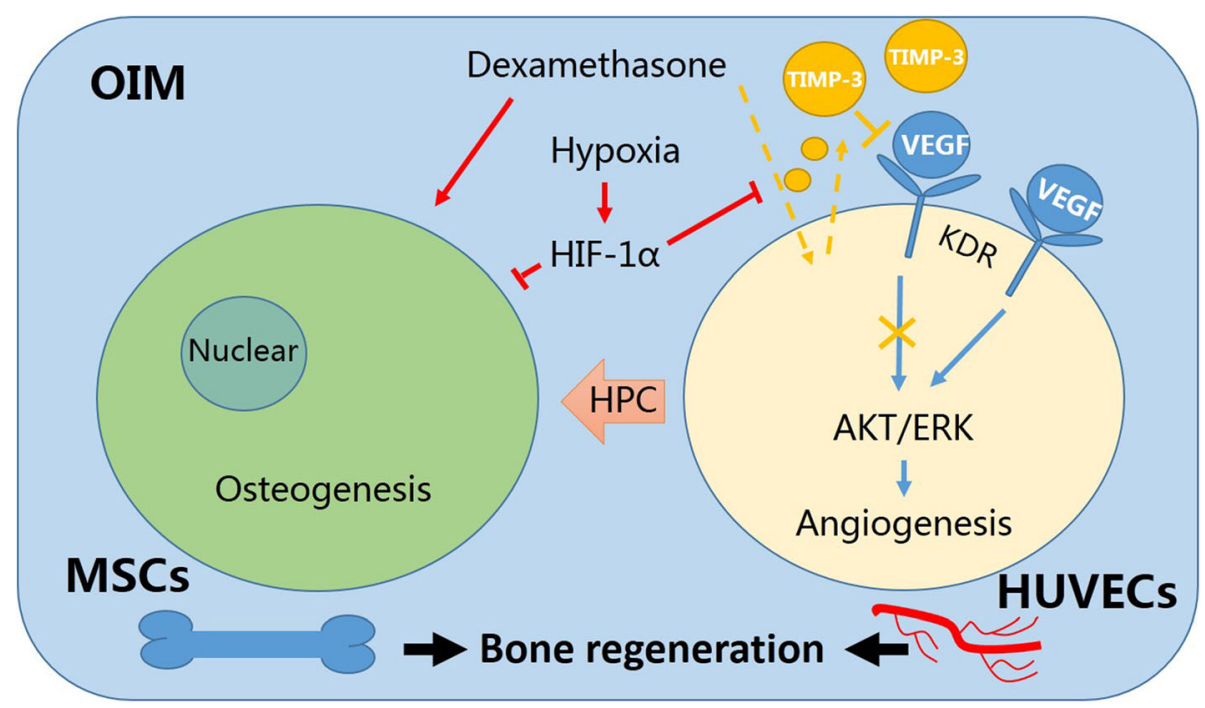

Fig. 8 Proposed mechanism by which hypoxia alleviates the dexamethasone-induced inhibition of angiogenesis in the coculture system with OIM

thus inducing cell proliferation and migration [28-30]. Similar to the results of other studies, the phosphorylation of KDR Y1175 residues was also very important for the formation of the vascular network structure in the present study. Inhibition of KDR phosphorylation reduces the proliferation and migration of HUVECs, and the vascular-like network structure will fail to form.

Dexamethasone can stimulate the expression of TIMP-3 in a variety of cells $[18,19]$. Traditionally, as a potential tumor suppressor gene, TIMP-3 specifically suppresses the activity of MMPs, leading to the inhibition of tumor invasion, angiogenesis and metastasis. In clinical trials, low TIMP-3 protein levels in patients are associated with poor prognosis in nonsmall cell lung cancer patients [31]. Recent studies have shown that TIMP-3 can compete with VEGF-A for binding to KDR, leading to the inhibition of blood vessel generation [32]. In tumor tissues, overexpression of TIMP-3 significantly reduces the vascular density of tumors, resulting in a smaller tumor volume [33]. The diterpenoid lactone andrographolide, the active compound of Andrographis paniculata, has antitumor activity. This compound inhibits miR-21-5p, promotes the expression of TIMP-3, prevents the phosphorylation of KDR and its downstream factor MAPK, and inhibits the proliferation and migration abilities of HUVECs, blocking tumor angiogenesis [34, 35]. In this study, increased expression of TIMP-3 after treatment with dexamethasone was also observed. The increased level of TIMP-3 in HUVECs reduced the phosphorylation of KDR, AKT, and ERK, thus inhibiting cell migration and the formation of a vascularlike network.

There are some methods to remedy dexamethasonemediated vascular depression. Dexamethasone harmed endochondral ossification by inhibiting angiogenesis in the chicken embryo model, and vascular inhibition could be reversed by the addition of insulin-like growth factor [36]. In the dexamethasone-induced rat femoral head necrosis model, vitamin $K_{2}$ improved the survival rate, migration, and angiogenesis of EAhy926 cells [37]. In a tumor model, dexamethasone decreased the expression of VEGF in tumor cells but did not affect the expression of VEGFR in HUVECs, and this inhibition could be reversed by hypoxia [38]. Similarly, in the present study, HIF- $1 \alpha$ induced by hypoxia reduced the expression of TIMP-3. It has also been reported that hypoxia can upregulate proangiogenic factors and downregulate antiangiogenic proteins, such as TIMP-3 and TIMP-1, also improving angiogenesis [20].

Cobalt ions are known to mimic hypoxia by artificially stabilizing the transcription factor HIF- $1 \alpha$. In this study, $\mathrm{CoCl}_{2}$ was used to alleviate vascular inhibition in the MSC-HUVEC coculture system. Previous experiments showed that $100 \mu \mathrm{M} \mathrm{CoCl}{ }_{2}$ had the best recovery effect on HUVEC angiogenesis, but it was found that OIM supplemented with $100 \mu \mathrm{M} \mathrm{CoCl}$ inhibited MCS osteogenesis. Therefore, HUVECs were pretreated with $50 \mu \mathrm{M}$ $\mathrm{CoCl}_{2}$ and then cocultured with MSCs in OIM. The effect of hypoxia on the osteogenesis of MSCs is still controversial. However, a large number of studies have shown that hypoxia or overexpression of HIF-1 $\alpha$ in cells is beneficial for MSC osteogenic differentiation [39, 40]. However, some studies have shown that hypoxia upregulates TWIS $\mathrm{T}$ expression through HIF- $1 \alpha$ and inhibits the expression of RUNX2, thereby inhibiting the osteogenic differentiation of MSCs [41]. The Notch signaling pathway is upregulated under hypoxic conditions to maintain cell stemness, which does not support MSC osteogenesis [42]. 
Similarly, in these studies, the results showed that $\mathrm{CoCl}_{2}$ was not conducive to MSC osteogenesis. However, highdensity oligonucleotide arrays indicated that genes induced by $\mathrm{CoCl}_{2}$ and hypoxia do not overlap in human hepatocellular carcinoma cells [43]. Therefore, it will be necessary to systematically evaluate the application of hypoxia in prevascularized bone tissue engineering.

\section{Conclusion}

OIM was found to inhibit the HUVEC angiogenesis in cocultures of MSCs and HUVECs in OIM. TIMP-3 expression was stimulated by dexamethasone and was identified as an angiostatic factor that competes with VEGF-A to bind to/with KDR, thus inhibiting the angiogenesis of HUVECs. Hypoxia downregulated TIMP-3 expression via HIF-1 $\alpha$. The coculture system of MSCs and hypoxia-preconditioned HUVECs showed stronger osteogenesis and angiogenesis. These results revealed the critical role of TIMP-3 in angiogenesis, and a hypoxia strategy was discovered for prevascularization in bone tissue engineering.

\section{Abbreviations}

BMSCs: Bone marrow-derived mesenchymal stem cells; HUVECs: Human umbilical vein endothelial cells; OIM: Osteogenic induction medium; DEXA: Dexamethasone; KDR (VEGFR2): Vascular endothelial growth factor receptor 2; TIMP-3: Tissue inhibitor of metalloproteinase; VEGF: Vascular endothelial growth factor; ERK: Extracellular regulated protein kinases; AKT (PKB): Protein kinase B; HIF-1a: Hypoxia-inducible factor-1a; DMEM: Dulbecco's modified Eagle's medium; $\beta$-gpd: $\beta$-Glycerophosphate disodium; L-Vc: L-Vitamin C; eNOS: Endothelial nitric oxide synthase; $\mathrm{CoCl}_{2}$ : Cobalt chloride; EGM: Endothelial cell growth medium supplemented with ECGS; ALP: Alkaline phosphatase; qRT-PCR: Quantitative real-time PCR; HPC HUVECs: Hypoxia-preconditioned HUVECS

\section{Acknowledgements}

Not applicable.

\section{Authors' contributions}

CMM performed all the experiments and wrote the manuscript. GC, SQH, LX, ZY, and JZY were involved in useful discussions during the development of this study and revised the manuscript. ZY and TWS contributed to the conception, design of the work or of parts of it, and its interpretation. The authors read and approved the final manuscript.

\section{Funding}

This work was supported by grants from the National Key Research and Development Program of China (Grant No. 2018YFC1105800), the National Natural Science Foundation of China (Grant No. 81671841), and the Fundamental Research Funds for the Central Universities (Grant No. 22221818014)

\section{Availability of data and materials}

The datasets used and/or analyzed during the current study are available from the corresponding author on reasonable request.

\section{Ethics approval and consent to participate}

All animal experiments in the research performed were approved by the Ethics Committee of East China University of Science and Technology.

\section{Consent for publication}

Not applicable.

\section{Competing interests}

The authors declare that they have no competing interests.
Received: 14 March 2020 Revised: 23 June 2020

Accepted: 24 July 2020 Published online: 06 August 2020

\section{References}

1. Mercado-Pagán ÁE, Stahl AM, Shanjani Y, Yang Y. Vascularization in bone tissue engineering constructs. Ann Biomed Eng. 2015;43(3):718-29.

2. Saran U, Gemini Piperni S, Chatterjee S. Role of angiogenesis in bone repair. Arch Biochem Biophys. 2014;561:109-17.

3. Cheng P, Li D, Gao Y, Cao T, Jiang H, Wang J, et al. Prevascularization promotes endogenous cell-mediated angiogenesis by upregulating the expression of fibrinogen and connective tissue growth factor in tissueengineered bone grafts. Stem Cell Res Ther. 2018;9(1):176.

4. Laschke MW, Menger MD. Prevascularization in tissue engineering: current concepts and future directions. Biotechnol Adv. 2016;34(2):112-21.

5. Chen W, Liu X, Chen Q, Bao C, Zhao L, Zhu Z, et al. Angiogenic and osteogenic regeneration in rats via calcium phosphate scaffold and endothelial cell co-culture with human bone marrow mesenchymal stem cells (MSCs), human umbilical cord MSCs, human induced pluripotent stem cell-derived MSCs and human embryonic stem cell-derived MSCs. J Tissue Eng Regen Med. 2018;12(1):191-203.

6. Lu L, Deegan A, Musa F, Xu T, Yang Y. The effects of biomimetically conjugated VEGF on osteogenesis and angiogenesis of MSCs (human and rat) and HUVECs co-culture models. Colloids Surfaces B-Biointerfaces. 2018; 167:550-9.

7. Li J, Ma Y, Teng R, Guan Q, Lang J, Fang J, et al. Transcriptional profiling reveals crosstalk between mesenchymal stem cells and endothelial cells promoting prevascularization by reciprocal mechanisms. Stem Cells Dev. 2015;24(5):610-23.

8. Fan $X$, Teng $Y, Y$, Z, Zhou Y, Tan WS. The effect of gap junction-mediated transfer of miR-200b on osteogenesis and angiogenesis in a co-culture of MSCs and HUVECs. J Cell Sci. 2018;131(13):jcs216135.

9. Scherzed A, Hackenberg S, Froelich K, Rak K, Schendzielorz P, Gehrke T, et al. The differentiation of hMSCs counteracts their migration capability and proangiogenic effects in vitro. Oncol Rep. 2016;35(1):219-26.

10. Shen Q, Fan X, Jiang M, Ye Z, Zhou Y, Tan WS. Inhibiting expression of Cxc19 promotes angiogenesis in MSCs-HUVECs co-culture. Arch Biochem Biophys. 2019;675:108108.

11. Hong JX, Liu J, Li LF, Han JL. Dexamethasone concentration and osteogenic differentiation of umbilical cord mesenchymal stem cells. Chinese J Tissue Eng Res. 2013;17(23):4204-11.

12. Sidiqi MH, Aljama MA, Bin Riaz I, Dispenzieri A, Muchtar E, Buadi FK, et al. Bortezomib, lenalidomide, and dexamethasone (VRd) followed by autologous stem cell transplant for multiple myeloma. Blood Cancer J. 2018; 8(11):106.

13. Ozmen A, Unek G, Korgun ET. Effect of glucocorticoids on mechanisms of placental angiogenesis. Placenta. 2017;52:41-8.

14. Aida K, Wang XL, Wang J, Li C, McDonald TJ, Nathanielsz PW. Effect of betamethasone administration to the pregnant baboon at 0.75 gestation on placental eNOS distribution and activity. Placenta. 2004;25(10):780-7.

15. Zha X, Sun B, Zhang R, Li C, Yan Z, Chen J. Regulatory effect of microRNA$34 \mathrm{a}$ on osteogenesis and angiogenesis in glucocorticoid-induced osteonecrosis of the femoral head. J Orthop Res. 2018;36(1):417-24.

16. Cenciarini M, Valentino M, Belia S, Sforna L, Rosa P, Ronchetti S, et al. Dexamethasone in glioblastoma multiforme therapy: mechanisms and controversies. Front Mol Neurosci. 2019;12:65.

17. Chen YY, Brown NJ, Jones R, Lewis CE, Mujamammi AH, Muthana M, et al. A peptide derived from TIMP-3 inhibits multiple angiogenic growth factor receptors and tumour growth and inflammatory arthritis in mice. Angiogenesis. 2014;17(1):207-19.

18. Leco KJ, Khokha R, Pavloff N, Hawkes SP, Edwards DR. Tissue inhibitor of metalloproteinases-3 (TIMP-3) is an extracellular matrix-associated protein with a distinctive pattern of expression in mouse cells and tissues. J Biol Chem. 1994;269(12):9352-60.

19. Nagar JK, Patel PP, Mohapatra JN, Sharma MM, Pandya GM, Umar MM, et al. Differential effects of dexamethasone and rosiglitazone in a sephadexinduced model of lung inflammation in rats: possible role of tissue inhibitor of metalloproteinase-3. Indian J Pharmacol. 2015:47(2):153-9.

20. Zhang J, Zhao J, Bai Y, Huang L, Yu W, Li X. Effects of p75 neurotrophin receptor on regulating hypoxia-induced angiogenic factors in retinal pigment epithelial cells. Mol Cell Biochem. 2014;398(1-2):123-34. 
21. Drager J, Harvey EJ, Barralet J. Hypoxia signalling manipulation for bone regeneration. Expert Rev Mol Med. 2015;17:e6.

22. Masoud GN, Li W. HIF-1alpha pathway: role, regulation and intervention for cancer therapy. Acta Pharm Sin B. 2015;5(5):378-89.

23. Gu C, Li P, Liu W, Zhou Y, Tan WS. The role of insulin in transdifferentiated hepatocyte proliferation and function in serum-free medium. J Cell Mol Med. 2019;23(6):4165-78.

24. Peach CJ, Mignone WW, Arruda MA, Alcobia DC, Hill SJ, Kilpatrick LE, et al. Molecular pharmacology of VEGF-A isoforms: binding and signalling at VEGFR2. Int J Mol Sci. 2018;19(4):1264.

25. Ong CS, Zhou J, Ong CN, Shen HM. Luteolin induces G1 arrest in human nasopharyngeal carcinoma cells via the Akt-GSK-3ß-Cyclin D1 pathway. Cancer Lett. 2010;298(2):167-75.

26. Zhou M, Shen S, Zhao X, Gong X. Luteoloside induces G0/G1 arrest and pro-death autophagy through the ROS-mediated AKT/mTOR/p70S6K signalling pathway in human non-small cell lung cancer cell lines. Biochem Biophys Res Commun. 2017:494(1-2):263-9.

27. Ma J, Guo X, Zhang J, Wu D, Hu X, Li J, et al. PTEN gene induces cell invasion and migration via regulating AKT/GSK-3 $/ \beta$-catenin signaling pathway in human gastric cancer. Dig Dis Sci. 2017;62(12):3415-25.

28. Wang $H$, Guan $Q$, Nan $Y, M a ~ Q$, Zhong Y. Overexpression of human MX2 gene suppresses cell proliferation, migration, and invasion via ERK/P38/NFKB pathway in glioblastoma cells. J Cell Biochem. 2019;120(11):18762-70.

29. Mao XH, Chen M, Wang Y, Cui PG, Liu SB, Xu ZY. MicroRNA-21 regulates the ERK/NF-KB signaling pathway to affect the proliferation, migration, and apoptosis of human melanoma A375 cells by targeting SPRY1, PDCD4, and PTEN. Mol Carcinog. 2017;56(3):886-94.

30. Zahradka P, Werner JP, Buhay S, Litchie B, Helwer G, Thomas S. NF-kB activation is essential for angiotensin II-dependent proliferation and migration of vascular smooth muscle cells. J Mole Cell Cardiol. 2002;34(12): 1609-21.

31. Xu C, Hou Z, Zhan P, Zhao W, Chang C, Zou J, et al. EZH2 regulates cancer cell migration through repressing TIMP-3 in non-small cell lung cancer. Med Oncol. 2013;30(4):713.

32. Qi JH, Ebrahem Q, Moore N, Murphy G, Claesson-Welsh L, Bond M, et al. A novel function for tissue inhibitor of metalloproteinases-3 (TIMP3): inhibition of angiogenesis by blockage of VEGF binding to VEGF receptor-2. Nat Med. 2003;9(4):407-15.

33. Qi JH, Ebrahem Q, Ali M, Cutler A, Bell B, Prayson N, et al. Tissue inhibitor of metalloproteinases-3 peptides inhibit angiogenesis and choroidal neovascularization in mice. PLoS One. 2013;8(3):e55667.

34. Dai J, Lin Y, Duan Y, Li Z, Zhou D, Chen W, et al. Andrographolide inhibits angiogenesis by inhibiting the Mir-21-5p/TIMP3 signaling pathway. Int J Biol Sci. 2017:13(5):660-8.

35. Shen K, Ji L, Lu B, Xu C, Gong C, Morahan G, et al. Andrographolide inhibits tumor angiogenesis via blocking VEGFANVEGRR2-MAPKs signaling cascade. Chem Biol Interact. 2014;218:99-106.

36. Xu S, Guo R, Li PZ, Li K, Yan Y, Chen J, et al. Dexamethasone interferes with osteoblasts formation during osteogenesis through altering IGF-1-mediated angiogenesis. J Cell Physiol. 2019; https://doi.org/10.1002/jcp.28157.

37. Zhang Y, Yin J, Ding H, Zhang C, Gao YS. Vitamin K2 ameliorates damage of blood vessels by glucocorticoid: a potential mechanism for its protective effects in glucocorticoid-induced osteonecrosis of the femoral head in a rat model. Int J Biol Sci. 2016;12(7):776-85.

38. Machein MR, Kullmer J, Rönicke V, Machein U, Krieq M, Damert A, et al. Differential downregulation of vascular endothelial growth factor by dexamethasone in normoxic and hypoxic rat glioma cells. Neuropathol Appl Neurobiol. 1999;25(2):104-12.

39. Wagegg M, Gaber T, Lohanatha FL, Hahne M, Strehl C, Fangradt M, et al. Hypoxia promotes osteogenesis but suppresses adipogenesis of human mesenchymal stromal cells in a hypoxia-inducible factor-1 dependent manner. PLoS One. 2012;7(9):e46483.

40. Zou D, Han W, You S, Ye D, Wang L, Wang S, et al. In vitro study of enhanced osteogenesis induced by HIF-1a-transduced bone marrow stem cells. Cell Prolif. 2011;44(3):234-43.

41. Milstone DS, Yang D-C, Yang M-H, Tsai C-C, Huang T-F, Chen Y-H, et al. Hypoxia inhibits osteogenesis in human mesenchymal stem cells through direct regulation of RUNX2 by TWIST. PLoS One. 2011;6(9):e23965.

42. Yang M, Liu H, Wang Y, Wu G, Qiu S, Liu C, et al. Hypoxia reduces the osteogenic differentiation of peripheral blood mesenchymal stem cells by upregulating Notch-1 expression. Connect Tissue Res. 2019;60(6):583-96.
43. Muñoz-Sánchez J, Chánez-Cárdenas ME. The use of cobalt chloride as a chemical hypoxia model. J Appl Toxicol. 2019;39(4):556-70.

\section{Publisher's Note}

Springer Nature remains neutral with regard to jurisdictional claims in published maps and institutional affiliations.

\section{Ready to submit your research? Choose BMC and benefit from:}

- fast, convenient online submission

- thorough peer review by experienced researchers in your field

- rapid publication on acceptance

- support for research data, including large and complex data types

- gold Open Access which fosters wider collaboration and increased citations

- maximum visibility for your research: over $100 \mathrm{M}$ website views per year

At BMC, research is always in progress.

Learn more biomedcentral.com/submissions 